\title{
Sinais específicos em Libras para o ensino odontológico
}

\author{
Lorena de Sousa Silva*; Jéssica Girlaine Guimarães Leal**; Gerson Ramalho Junior***; Marco \\ Antônio Dias da Silva****; Andresa Costa Pereira****
}
* Cirurgiã dentista, Curso de Odontologia, Universidade Federal de Campina Grande, campus de Patos
** Docente, Curso de Licenciatura Plena em Letras Libras, Universidade Federal Rural do Semiárido, campus Caraúbas
*** Docente de Libras, Universidade Federal de Campina Grande, campus de Patos
**** Docente, Curso de Odontologia, Universidade Federal de Campina Grande, campus de Patos

Recebido em 18/09/2017. Aprovado em 09/01/2018.

\begin{abstract}
RESUMO
Diversas iniciativas governamentais têm sido realizadas para promover a inclusão de pessoas surdas no ensino superior. Entretanto, o ingresso de um estudante surdo em um curso de Odontologia é de extrema complexidade, devido a vários motivos, dentre eles a carência de termos odontológicos em Libras. O objetivo deste trabalho foi criar e disponibilizar sinais odontológicos específicos em Libras. Uma equipe multidisciplinar selecionou os quinze primeiros termos a serem criados, os quais tiveram seus conceitos e explicações técnicas demonstrados para um docente surdo. Em seguida, o professor criava o sinal, que era registrado por vídeos e fotografias. Os dados técnicos da sinalização foram descritos detalhadamente e em seguida publicados junto às fotos e vídeos em sítio web. A criação do site e a disponibilização dos termos odontológicos específicos em Libras caracteriza o início de um processo de inclusão e permanência de pessoas surdas no Curso de Odontologia. A continuidade desse trabalho permitirá que os sinais sejam constantemente criados e adicionados ao glossário, a fim de auxiliar o ensino de pessoas surdas e aperfeiçoar a atuação do tradutor/interprete de Libras, contribuindo de forma pioneira para a formação de futuros cirurgiões dentistas surdos.

Descritores: Odontologia. Linguagem de Sinais. Inclusão Educacional. Instituições Acadêmicas.
\end{abstract}

\section{INTRODUÇÃO}

Nos últimos anos, a educação brasileira tem sofrido transformações importantes no processo de inclusão de indivíduos surdos, principalmente após a aprovação da lei
$10.436 / 2002$ e do decreto $5.626 / 2005^{1,2}$. Na lei 10.436, reconheceu-se a Língua Brasileira de Sinais (Libras) como meio legal de expressão e comunicação $^{3}$, enquanto no decreto 5.626 determinou-se que as instituições de ensino 
devem garantir acesso às pessoas surdas desde a educação infantil até a educação superior ${ }^{4}$.

Apesar de iniciativas importantes das Instituições de Ensino Superior (IES) na tentativa de adequação a essa nova realidade, destaca-se que ainda existem sérias deficiências que impedem o ingresso ou a permanência do estudante surdo no sistema ${ }^{5,6}$. Entende-se que as IES ainda necessitam se adaptar para proporcionar condições ideais de ensinoaprendizagem para estes discentes ${ }^{7,8}$.

$\mathrm{Na}$ universidade, nota-se que a maioria dos estudantes e docentes é ouvinte e os docentes desconhecem as peculiaridades da surdez, além de não dominarem a Libras, utilizando a língua portuguesa que é o idioma predominante na educação brasileira ${ }^{7}$.

Para a inclusão do surdo no ambiente universitário, a presença de tradutor/intérprete de Libras é essencial, entretanto não é suficiente ${ }^{7,8}$. A ausência de termos técnicos específicos em Libras gera um grande problema para o intérprete ${ }^{9}$.

A inserção de uma disciplina de Libras no Projeto Pedagógico de cursos de Odontologia já é realidade ${ }^{10,11}$ e esse processo ressalta a preocupação em formar cirurgiões dentistas preparados para o atendimento ao paciente surdo, efetivando a inclusão no serviço odontológico ${ }^{12-}$ 15. Este objetivo social é indispensável na formação do cirurgião dentista brasileiro. Entretanto, pouco se tem realizado no sentido de criar um ambiente educacional acolhedor para o ingresso do aluno surdo nos cursos de Odontologia.

Os conteúdos odontológicos em Libras são escassos e majoritariamente voltados para a atenção ao paciente. Esse fato ressalta a necessidade de se criar termos novos e específicos, disponibilizando-os de forma que possam ser facilmente encontrados ${ }^{16}$.

Buscando suprir essa carência, os autores se propuseram a identificar e criar sinais odontológicos específicos em Libras, divulgando-os em um sítio web desenvolvido para esse fim, acessível ao estudante surdo, tradutor/intérprete e/ou professor de qualquer curso de Odontologia do Brasil.

\section{METODOLOGIA}

O presente trabalho foi realizado por uma equipe multidisciplinar composta por docente $e$ acadêmica do curso de Odontologia, tradutor/interprete e professor de Libras membro da comunidade surda. $\mathrm{O}$ estudo foi dividido em quatro etapas principais: pesquisa e seleção de termos odontológicos, criação dos sinais em Libras para os termos selecionados, registro dos sinais criados por meio de fotos e vídeos, e elaboração de materiais didáticos de apoio ao ensino.

A etapa de pesquisa dos termos odontológicos foi realizada pela consulta em um dos principais dicionários em Libras ${ }^{17}$, pela busca online de artigos nas bases SciELO e PubMed, além de consultas à comunidade surda de várias instituições do país. Frente à escassez de termos odontológicos já existentes, optou-se por iniciar a criação dos sinais definindo as áreas/especialidades de atuação do cirurgião dentista, presentes nas disciplinas acadêmicas do curso de Odontologia e cadastradas pelo Conselho Federal de Odontologia (CFO).

Os termos definidos foram Cirurgia e Traumatologia Bucomaxilofacial, Dentística, Disfunção Temporomandibular, Endodontia, Estomatologia, Implantodontia, Odontogeriatria, Odontologia para Pacientes com Necessidades Especiais, Odontopediatria, Ortodontia, Patologia Bucal, Periodontia, Prótese Dentária, Radiologia Odontológica e Imaginologia, e Saúde Coletiva e da Família.

Os sinais foram criados pelo autor professor de Libras (GRJ), membro da 
comunidade surda. Inicialmente, a equipe de Odontologia, com o auxílio da intérprete, realizava a explanação para cada termo escolhido, conceituando-o e apresentando vídeos explicativos e textos.

Em seguida, o professor surdo realizava a sinalização, caracterizada pelos cinco parâmetros básicos na formação de um sinal em Libras (configuração de mãos, ponto de articulação, movimento, orientação e expressões não manuais). A configuração de mãos era determinada pelo número correspondente à posição da mão, como proposto por Felipe e Lira $(2005)^{18}$. O ponto de articulação era definido segundo o espaço ou local do corpo onde era realizado, seguindo o padrão de Ferreira-Brito $(1995)^{19}$. Já os movimentos e as orientações das mãos durante a sinalização foram descritos detalhadamente e colocados em quadros. O último parâmetro de expressões não manuais foi caracterizado pelas expressões faciais e corporais realizadas durante a sinalização de cada termo.

Para o registro de todos os sinais criados, a sinalização era fotografada e filmada de forma padronizada, com distância aproximada de 1,5 metros, utilizando padrões de roupa escura do professor e fundo claro.

A etapa de elaboração do material didático de apoio ocorreu com a criação de quadros informativos contendo as fotos da execução do sinal e detalhamento sobre as configurações de mãos, os pontos de articulação, movimentos, orientações e expressões não manuais de cada termo.

O material em vídeo foi editado, se inseriu legenda e alterou as configurações de sons. Todo o material desenvolvido foi então disponibilizado em um sítio web criado especificamente para esse fim (http://revistas.ufcg.edu.br/odontologiaemlibras). Optou-se por uma estrutura simples, com o objetivo de manter o fácil acesso ao conteúdo completo dos sinais criados. Os termos disponibilizados podem ser encontrados em ordem alfabética pelo glossário ou pelas áreas odontológicas específicas. Para cada termo, disponibilizou-se link para a sinalização por vídeo ou por fotos.

\section{RESULTADOS}

A figura 1 exibe a página do sítio web criado, com os conteúdos disponibilizados de acordo com as áreas odontológicas. Para cada termo é possível visualizar a sinalização por vídeo ou por fotos.

Ao clicar em sinalização por vídeo exibese a página representada na figura 2, na qual é possível assistir à sinalização do termo.

$\mathrm{Na}$ exibição da sinalização por fotos, é exposto o quadro 1, o qual não apenas apresenta as imagens registradas, mas também 0 detalhamento de cada sinalização de acordo com os cinco parâmetros básicos em Libras.

\section{DISCUSSÃO}

No presente trabalho criou-se, de forma inédita, material que permite a inclusão do aluno surdo no ensino superior de Odontologia. Sabese que por se encontrar em fase de pesquisa muito recente, a Libras ainda possui grande escassez de termos profissionais específicos, sendo que algumas iniciativas recentes têm sido referenciadas pela confecção de sinais e materiais de apoio ao aluno surdo em diversas áreas, como $\operatorname{Artes}^{20}$, Química ${ }^{21}$, Letras Libras $^{22}$, Música ${ }^{23,24}$, Desenho Arquitetônico 9 , Engenharia de Produção ${ }^{25}$, Matemática ${ }^{26}$, Física $^{27}$ Biologia ${ }^{28}$ Engenharia $\mathrm{Civil}^{29}$. Entretanto, nenhum projeto semelhante foi encontrado no ensino superior na Área da Saúde.

A carência deste tipo de publicação pode ser justificada por implicar em processo cuidadoso para a criação de novos sinais. Miranda et al. $(2017)^{30}$ relatam que não concluíram a produção de sinais que propuseram devido à dificuldade e 
tempo requeridos neste processo, sendo odontológicos criados no presente trabalho foram indispensável que o sinal seja aceito pela debatidos e aprovados em evento com a presença comunidade surda ${ }^{25}$. Neste sentido, os termos de professores de Libras e da comunidade surda.

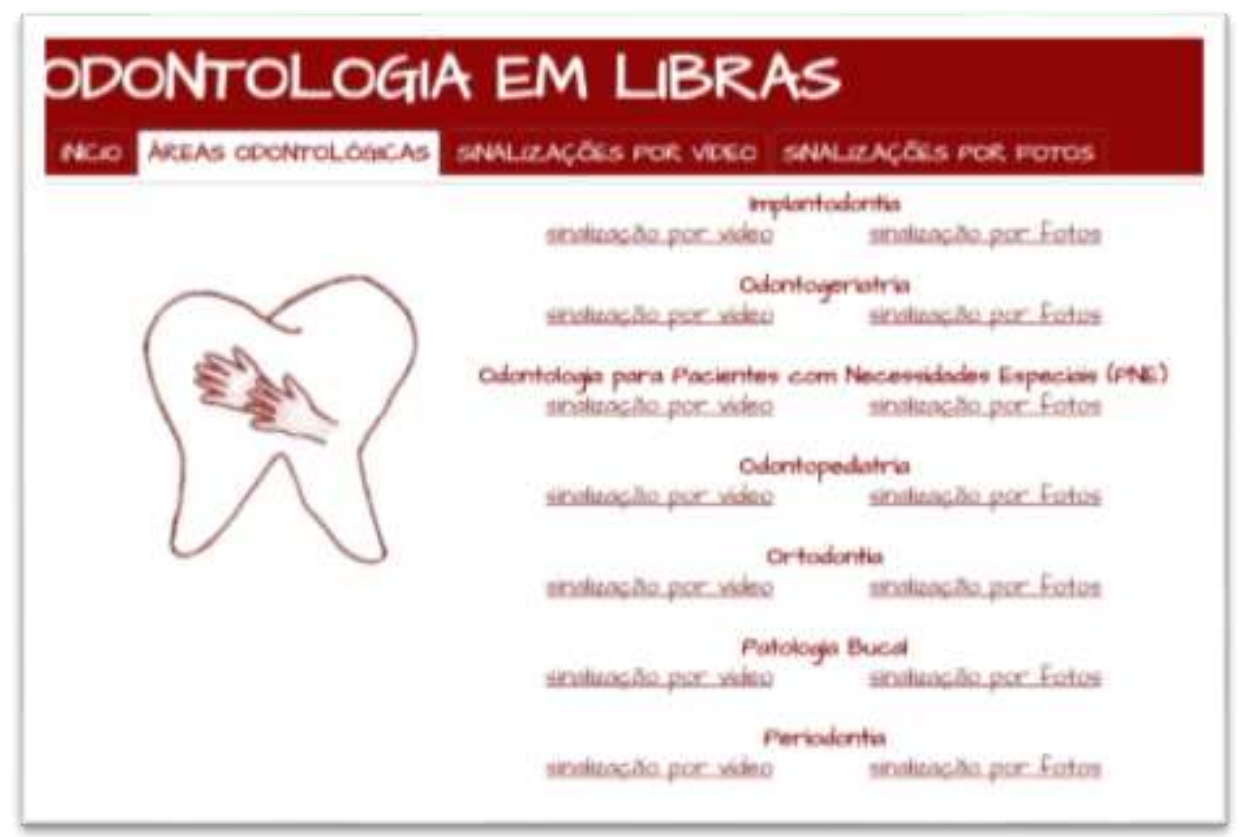

Figura 1. Tela do sítio web contendo os termos odontológicos criados

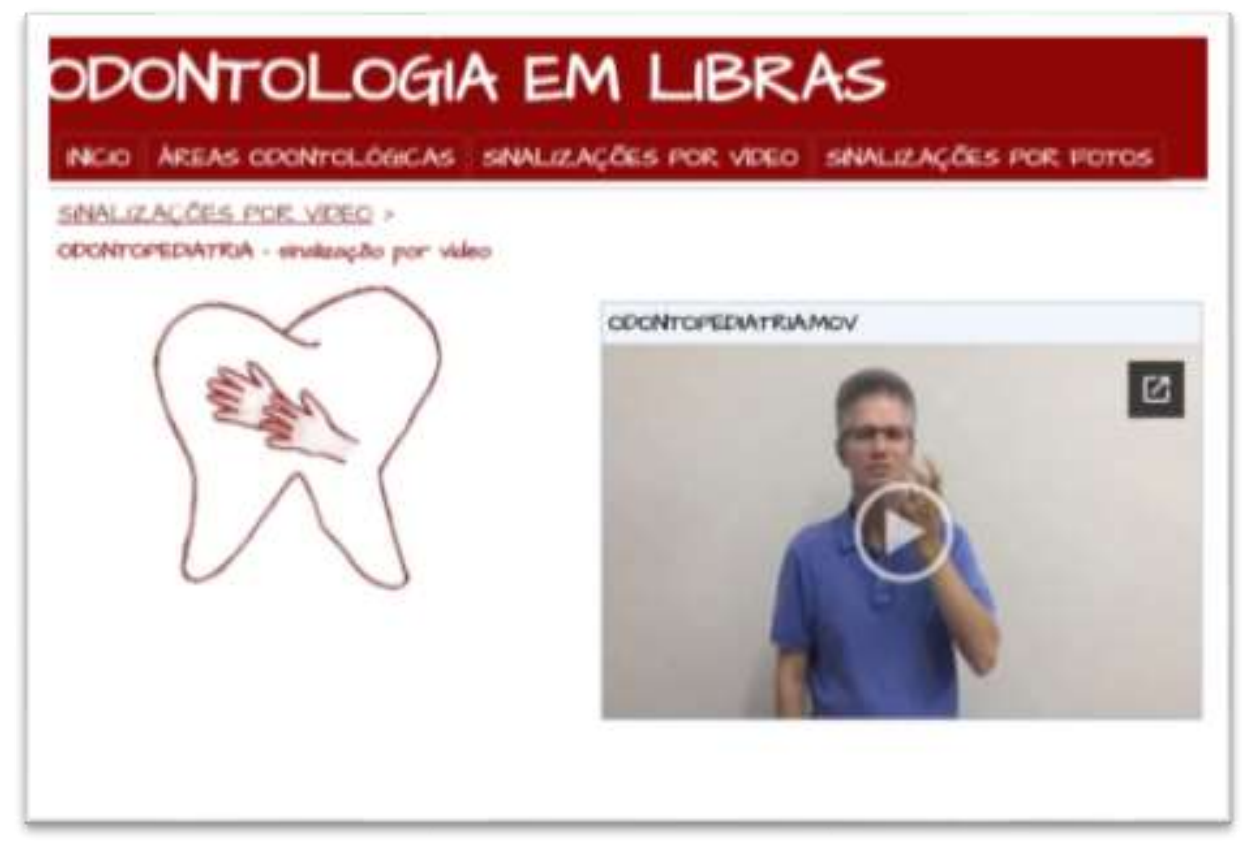

Figura 2. Imagem da página de exibição da sinalização por vídeo do termo Odontopediatria 
Quadro 1. Termo Odontopediatria sinalizado por fotos, além da descrição da configuração de mãos, ponto de articulação, movimento, orientação e expressão não manual

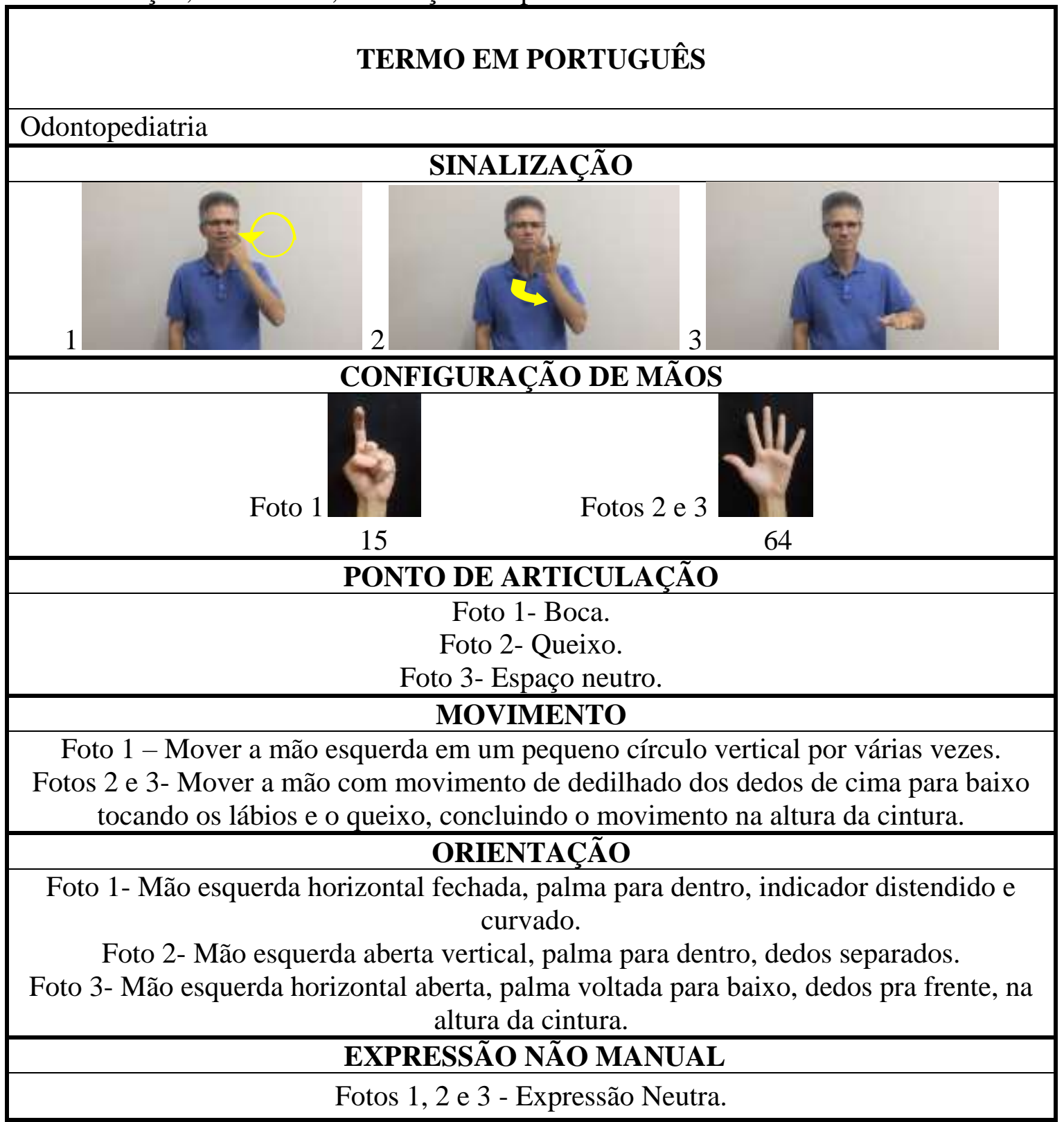

Já a metodologia de registro da sinalização em vídeo também se assemelha à descrita na literatura $^{25,28}$, incluindo as etapas de explicação detalhada sobre o tema para o membro da comunidade surda, que em seguida cria o sinal e o executa para registro em vídeo. Em relação à montagem dos quadros detalhados de cada termo criado, seguimos a observação realizada por Lima $(2014)^{9}$, ressaltando a importância de uma ficha léxico-terminográfica, devido à intensidade de informações que as mesmas representam. Os quadros do presente trabalho reproduzem as fotos da sinalização, termo em português e caracterização de acordo com os cinco parâmetros e são semelhantes aos criados por Kuhn $(2014)^{25}$. Por outro lado, estão mais 
simples que a ficha léxico-terminográfica detalhada por Lima (2014) ${ }^{9}$, a qual disponibiliza informações adicionais, dentre elas a definição do termo, a exemplificação da utilização do termo em uma frase e a representação do sinal em sua forma escrita (SignWriting). A escrita de sinais, também encontrada no glossário de Oliveira e Stumpf $(2013)^{22}$ pode ser uma sugestão de complementação futura do conteúdo online produzido o presente estudo.

Um fator extremamente positivo proposto neste trabalho é que a padronização da produção de todos os sinais (com critérios para a sua criação pelo surdo, registro dos vídeos, das fotos e dos conteúdos em quadro) permite que diversos grupos acadêmicos nacionais possam integrar a equipe executora deste projeto, trabalhando juntos apesar da distância física, mas integrados pelas tecnologias de informação e comunicação.

Seguindo o trabalho realizado por Lima $(2014)^{9}$, optou-se por criar um material digital online, levando em consideração que a internet é caracterizada como uma boa ferramenta para o ensino-aprendizagem em Libras ${ }^{31}$. Concordamos com Vales $(2008)^{20}$, Sagário et al. $(2012)^{16}$ e Lima $(2014)^{9}$ quando os mesmos ressaltam não apenas a necessidade da criação, mas também de divulgação dos novos termos, para amplo acesso aos materiais. O sítio web permite o acesso de qualquer indivíduo interessado em conhecer termos odontológicos específicos e contribui para a expansão do léxico e difusão da Libras. Essa forma de apresentação do material permite uma maior dinâmica das informações, possibilitando que novos termos criados possam ser adicionados a qualquer momento. Esse fator é bastante relevante para o presente projeto, que busca ter uma contribuição contínua, colaborativa e com repercussão nacional, integrando os cursos de Odontologia. Ademais, a cooperação entre IES torna o projeto mais amplo e permite atingir de forma mais homogênea os cursos de todas as regiões do Brasil.

Até o momento, os poucos sinais odontológicos referenciados na literatura são termos gerais em saúde bucal, que podem ser utilizados principalmente no atendimento ao paciente surdo ${ }^{16}$. Indiscutivelmente, esses sinais são insuficientes para um estudante surdo conseguir concluir um curso de Odontologia e desta forma a criação dos termos técnicos específicos se torna indispensável.

A verdadeira inclusão dos surdos no ensino superior de qualidade permite aos mesmos demonstrar a capacidade de se tornarem bons profissionais $^{25}$. De acordo com o decreto $5.626 / 2005$, as IES devem garantir o ensino superior às pessoas surdas, além de oferecer o auxílio profissional de um tradutor e intérprete de Libras ${ }^{4}$. Assim, além do nosso trabalho de criação de sinais odontológicos como ferramentas educacionais, é necessário que as instituições adequem estrutura física, insiram recursos tecnológicos e profissionais qualificados, com a presença de tradutor/interprete de Libras e professores capacitados $^{2,32}$.

\section{CONSIDERAÇÕES FINAIS}

A disponibilização de termos odontológicos específicos em Libras caracteriza o início de um processo de inclusão e permanência de pessoas surdas nos cursos de Odontologia. A continuidade desse trabalho permitirá que os sinais sejam constantemente criados e adicionados ao glossário, a fim de auxiliar o ensino de pessoas surdas e aperfeiçoar a atuação do tradutor/interprete de Libras.

As tecnologias permitem ampliar o alcance do trabalho e os termos podem ser acessados, a qualquer momento e local, pelo aluno surdo, pelo tradutor/intérprete, pelo professor ou qualquer profissional/estudante interessado no assunto, contribuindo de forma pioneira para formação de 
futuros cirurgiões dentistas surdos.

\section{ABSTRACT \\ Specific terms in Brazilian sign language for dental education}

Several governmental initiatives have been undertaken to include deaf people in higher education. However, the admission of a deaf student in the School of Dentistry has several difficulties, including the lack of dental signs in Brazilian sign language (Libras). The aim of this work was to create and make available specific dental signs in Libras. Then the teacher created the signal, which was recorded by videos and photographs. The technical data of the signs were described in detail and then published together with the photos and videos on the website. The creation of the website and the availability of the specific dental terms in Libras characterizes the beginning of a process of inclusion and permanence of deaf people in the Dental courses. The continuity of this work will allow the signs to be constantly created and added to the glossary in order to assist the teaching of deaf people and improve the translator/interpreter performance of Libras, contributing in a pioneering way to the training of future deaf dental surgeons.

Descriptors: Dentistry. Sign language. Educational Inclusion. Academic Institutions.

\section{REFERÊNCIAS}

1. Giroto CRM, Martins SESO, Lima JMR. Inserção da disciplina libras no ensino superior. J Res Spec Educ Needs. 2016; 16(1):662-5.

2. Santana AP. A inclusão do surdo no ensino superior no Brasil. J Res Spec Educ Needs. 2016;16(1):85-8.

3. Brasil. Presidência da República. Casa Civil. Subchefia para assuntos Jurídicos. Lei 10.436 de 24 de abril de 2002, que dispõe sobre a Língua Brasileira de Sinais - Libras e dá outras providências [Internet]. Diário Oficial [da] União. Brasília, DF; 2002 [Acesso em 18/06/2017]. Disponível em: http://www.planalto.gov.br/ccivil_03/leis/2 002/110436.htm

4. Brasil. Presidência da República. Casa Civil. Subchefia para Assuntos Jurídicos. Decreto $\mathrm{n}^{\mathrm{o}}$ 5.626, de 22 de dezembro de 2005. Regulamenta a Lei no 10.436, de 24 de abril de 2002, que dispõe sobre a Língua Brasileira de Sinais - Libras, e o art. 18 da Lei no 10.098, de 19 de dezembro de 2000. Diário Oficial [da] União [Acesso em 18/06/2017]. Disponível em: http://www. planalto.gov.br/ccivil_03/_ato20042006/20 05/decreto/d5626.htm

5. Moreira LC, Ansay NN, Fernandes SF. Políticas de acesso e permanência para estudantes surdos ao ensino superior. Rev Teor Prát Educ. 2016;19(1):49-60.

6. Rocha LRM, Santos LF. O que dizem os estudantes surdos da Universidade Federal de Santa Maria sobre a sua permanência no ensino superior. Práxis Educativa. 2017; 12(3):1-22.

7. Bisol CA, Valentini CB, Simioni JL, Zanchin J. Estudantes surdos no ensino superior: reflexões sobre a inclusão. Cad Pesqui. 2010; 40(139):147-72.

8. Daroque SC. Alunos surdos no ensino superior: uma discussão necessária. [Dissertação]. Piracicaba: Universidade Metodista de Piracicaba; 2011.

9. Lima VLS. Língua de sinais: proposta terminológica para a área de desenho arquitetônico. [Tese]. Belo Horizonte: Universidade Federal de Minas Gerais; 2014.

10. Oliveira YCA, Costa GMC, Coura AS, Cartaxo RO, França ISX. A língua brasileira de sinais na formação dos profissionais de Enfermagem, Fisioterapia e Odontologia no estado da Paraíba, Brasil. Interface (Botucatu). 2012; 16(43):973-86.

11. Ramos TS, Almeida MAPT. A importância 
do ensino de Libras: relevância para profissionais de saúde. Id on Line. 2017; 10(33):116-26.

12. Pereira RM. Percepção das pessoas surdas sobre o processo de comunicação no atendimento odontológico. [Graduação]. Natal: Universidade Federal do Rio Grande do Norte; 2015.

13. Silva MC, Rodrigues WE. Acessibilidade no tratamento odontológico do paciente surdo. Rev CROMG. 2015; 16(1):12-8.

14. Mendonça DS. Atendimento odontológico ao surdo. [Graduação]. Porto Velho: Faculdade São Lucas; 2015.

15. Esmeraldo MRA. Percepção de surdos que receberam informações sobre saúde bucal na linguagem oral e na linguagem de libras. [Tese]. São Paulo: Universidade Cruzeiro do Sul; 2015.

16. Sagário J, Gomes MPV, Botelho MPJ. Uma proposta para melhorar a comunicação entre profissionais de odontologia e o paciente surdo. In: Anais da $6^{\mathbf{a}}$ mostra interna de trabalhos de iniciação científica, 2012. p. 114.

17. Capovilla FC, Raphael WD. Dicionário enciclopédico ilustrado trilíngue da língua brasileira de sinais. São Paulo: Editora da Universidade de São Paulo, 2001.

18. Felipe TA, Lira GA. Dicionário da Língua Brasileira de Sinais - Libras. Rio de Janeiro, Acessibilidade Brasil - CORDE. Versão 2.0, 2005.

19. Ferreira-Brito L. Por uma gramática de língua de sinais. Tempo Brasileiro UFRJ. Rio de Janeiro, 1995.

20. Vales LS. Pequeno dicionário regional de Libras para artes. [Especialização]. Porto Alegre: Universidade Federal do Rio Grande do Sul; 2008.

21. Sousa SF, Silveira HE. Terminologias Químicas em Libras: A utilização de sinais na aprendizagem de alunos surdos. Química Nova na Escola, 2011; 33(1):37-46.

22. Oliveira JS, Stumpf MR. Desenvolvimento de glossário de Sinais Acadêmicos em ambiente virtual de aprendizagem do curso Letras-Libras. Informática na Educação: teoria e prática. 2013; 16(2):217-228.

23. Ribeiro DP. Glossário bilíngue da língua de sinais brasileira: criação de sinais dos termos da música. [Dissertação]. Brasília: Universidade de Brasília; 2013.

24. Benassi CA, Duarte AS. Além dos sentidos: glossário de termos e conceitos da área musical em Libras. Rev Diálogos. 2016; 4(1):9-25.

25. Kuhn TCG. Processo de criação de termos técnicos em libras para engenharia de produção. [Dissertação]. Ponta Grossa: Universidade Tecnológica Federal do Paraná; 2014.

26. Moreira FSR. A língua de sinais brasileira (Libras) na educação de surdos uma proposta para a elaboração de glossário de matemática. In: Trabalhos do $6^{\circ}$ EBREM, Brasília, 2014. p. 1-11.

27. Vargas JS, Gobara ST. Elaboração e utilização de Sinais de Libras para os conceitos de Física: Aceleração, Massa e Força. R Bras Ensi Ci Tecnol. 2015; 8(2):129-44.

28. Dantas MM, Pereira PTS, Leite RCL, Carvalho LCM, Onofre E. Criação de sinais libras biológicos para o conteúdo de membrana plasmática. In: Anais do $2^{\circ}$ CINTEDI, Campina Grande, 2016. p. 1-9.

29. Garcia KFL, Silva TA, Silva TA, Junior IQS. Sinais específico em libras: curso técnico em edificações e superior em engenharia civil. In: Resumos expandidos da $13^{\text {a }}$ semana de licenciatura. 2016. p. 396401.

30. Miranda IM, Mourão VLA, Gediel ALB. As 
tecnologias da informação e comunicação (TICs) e os desafios da inclusão: a criação de aulas sinalizadas no contexto do ensino superior. Periferia. 2017; 9(1):243-62.

31. Gediel ALB, Soares CP, Oliveira CLR. O ambiente virtual como aliado no processo de ensino e aprendizagem da Libras. (Con) Textos Linguísticos. 2016; 10(16):24-37.

32. Omote $\mathrm{S}$. Atitudes em relação à inclusão no ensino superior. J Res Spec Educ Needs. 2016; 16(1):211-5.

\section{Correspondência para:}

Andresa Costa Pereira

e-mail: andresa@cstr.ufcg.edu.br

Curso de Odontologia - UACB/CSTR

Universidade Federal de Campina Grande

Av. dos Universitários, S/N

Rodovia Patos/Texeira, Km 1, Santa Cecília 58708-110 Patos/PB 